\title{
Interactive comment on "Densified multi-mission observations by developed optical water levels show marked increases in lake water storage and overflow floods on the Tibetan Plateau" by Xingdong Li et al.
}

\section{Anonymous Referee \#2}

Received and published: 27 May 2019

This study combines altimetry data that measure lake levels directly with shoreline positions from optical data to create extended and denser lake level time series for the largest lakes of the TP. In that sense, the resulting dataset differs from existing lake level time series and seems thus a valuable resource for the scientific community as well as other users. The study is relevant for ESSD and worth publishing. To properly document the data and methods and to comply with ESSD's guidelines, the manuscript needs to be improved - in particular to better describe important parts of the methods, include/consider uncertainties, and properly validate the time series against existing 


\section{General comments}

1) The study would benefit from a clearer story line and justification how this work/data fills a current knowledge gap. I only understood the plot halfway through the methods. What are the shortcomings of the existing studies/datasets, and how do you overcome these with your study? This is especially important for the introduction, but also the abstract and conclusion would benefit from an easier to understand quick summary. See also comment paragraph P8, L11ff below.

2) Method: the important novelty of your approach is the use of shoreline positions from optical data to increase the temporal resolution and extend the length of the water level time series. To do so, you relate shoreline positions to lake level elevations from spaceborne altimetry data, using a statistical relationship between the two. Currently, the statistics part is not well enough described, and uncertainties from the found relationship do not seem to be propagated to your final "optical water levels". I suggest you extend this part to provide more transparency and include also a discussion of the uncertainties, considering in particular the assumption of a linear relationship (?) and whether it is appropriate to extrapolate beyond the range of measured lake levels.

3) Dataset: I'm missing a detailed description of the final dataset and its attributes and uncertainties, e.g. after the validation section.

4) Validation (and uncertainties):

- What is the accuracy/uncertainty of the altimetry products, and how does this propagate to your optical water levels? Consider also the uncertainty of the statistical relationship(s) you compute to derive the optical water levels. 
- The theoretical computation of an uncertainty (most of 4.2) based on a single UAV image is not convincing to me as it is based on a single image pair only with unknown coregistration accuracy (see comment below). The lack of hands-on data basis and the extensive length of the theoretical part makes this off-topic. Maybe this could fit as supplementary information in a separate document.

- Rather than treating the comparison to the LEGOS Hydroweb data as an application case this should be part of the validation section. How do your time series compare to the other datasets listed in table 1 ?

For data description, uncertainties and validation see ESSD's guidlines at https://www.earth-syst-sci-data.net/10/2275/2018/, in particular sections 3.3, 3.5 and 3.6.

\section{Specific comments}

A simpler title might make it easier to understand what the study is about. Especially the rather unclear terms "densified" and "developed optical water levels" should be replaced. Focus on the data and not the application cases.

\section{Abstract}

The abstract could be more to the point. Add some information on the performance of your data (uncertainties and validation). Consider removing already published findings (applications).

L12: which altimetric missions? If there are too many to list all, specify how many and which types (e.g. lidar altimetry, interferometric SAR altimetry...) 
L13: avoid putting important information in brackets. Monthly to weekly time series?

L16: "partial altimetry data" and "optical water levels" are unclear terms

L19: "densified" is unclear

L20ff: Are these groundbreaking new numbers/findings? Consider removing them and focus on the dataset.

Interactive

comment

Introduction

P2, L3: A strong motivation for TP lake studies not mentioned here is to find out why they are expanding, i.e. a good data set will contribute to a better understanding of climate and circulation patterns and changes thereof. This is important as the TP has a strong influence on both regional climate.

P2, L6: source of that number?

P2, L8: I wonder why you selected exactly these references? There are many more lake studies on the TP. References for the method (general) and local application should be separated.

P2, L11: It is better to introduce radar and lidar separately as the systems and data are quite different. Also, these data are not meant for ice berg height - you probably mean ice sheet surface elevation or sea ice freeboard?

P2, L16: The satellite is called ICESat, not ICESat-1. Change everywhere.

P2, L25: it seems you mainly mean (and in your study only use) optical data. Do you have an example for a sensor and study that used SAR data?

P2, L26: why exactly these references? These are not the only or first such studies.

P2, L33: references for the water index and Otsu algorithm? 
P3, L10f: remaining bias: is this not true for your study, too? Or how do you avoid/remove such bias?

P3, table 1: Does this table include all studies, or how did you select? Either remove all that don't compute lake levels, otherwise consider also including "complete" TP water studies for a larger number of lakes than the ones you are listing (e.g. Pekel et al (2016) to whom you refer to earlier, or Yang et al. 2019, doi:10.5194/tc-2018-238; Treichler et al. 2018, doi:10.5194/tc-2018-238...)

P4, L4: the meaning of "hypsometric curve" is unclear to me in this context.

Study area and data

Parts of this (e.g. from P5, L24, or P6, L1ff) rather belongs to the method section.

P4, L16: "as opposed to many other places..." - I tend to disagree, as nearly all seem to have expanded. Can you justify or explain more clearly?

P5, L4ff: why did you choose these lakes in particular? And where is Lake Yamzhog Yumco? An overview map might be useful.

P5, L17: "moderate set of orbital parameters" is unclear

P5, L30: when were the drone data acquisitions?

P5, L31: "similar" in what sense? What have Huang et al done?

P6, table 2: Some of the missions included many instruments (e.g. ENVISAT: 10 sensors). You need to specify which sensor and data you used. Here, you distinguish between "radar" and "interferometer", which is also based on radar (SAR/interferometric radar altimeter). This is confusing, and it would be useful to explain the technologies/differences either in the introduction or in a separate paragraph in the data or methods section. 
The first paragraph seems to explain what this study is about and would thus fit (better?) to the introduction (it is missing there!).

P6, L8: "comparing the mean water level of the overlap period" is vague. Explain better.

P7, figure 1: refer to the figure in the text, e.g. when you introduce the data and where comment you are talking about overlap periods. Consider adding the optical data to the figure to show the overlap periods you use to create the optical lake level - lake surface elevation relationship.

P7, L18: It is very unclear what "ENVISAT product" you used.

P7, L23: "highest bucket" is an unclear term. What elevation bin spacing did you choose for your frequency histograms? It seems you are losing information by binning your surface elevation measurements. How does that affect the accuracy of the extracted lake level elevations? I assume you have t-distributed data, i.e. roughly bell-shaped elevation distributions with long tails. It might be more appropriate to use the median elevation measurement, maybe in combination with a threshold to remove biased measurements in the tails. From reference DEMs, you should know the true surface elevation (of the lake shore).

P8, L4ff: How large are the biases you found? Are they constant over time and in space? I assume you compute this per lake?

P8, L13: it is unclear what you mean with "merging using optical water levels"

paragraph P8, L11ff: Only after reading this paragraph I think I finally understood the purpose of this study: You want to generate continuous lake level (volume?) series for as many lakes as possible. This requires elevation (and areal?) data from different sensors, as missions only last for a few years. As an additional challenge, the satellites in question have different orbits that only cover some lakes each, so not all elevation 
datasets can be used for each lake. For each lake, you therefore combine lake level elevation time series from the different sensors with data for that lake, using the overlap periods to correctly align the records, i.e. you remove potential elevation bias between the time series and make sure they are consistent. Where there is no sufficient overlap, you use optical data as a proxy: you create a statistical relationship between lake levels (from altimetry data) and corresponding shoreline position (from optical data acquired at the same time), and then apply (extrapolate?) the relationship to (optical) shoreline positions for time periods where you lack surface elevation data, but do have optical data. I propose you add something like this to the introduction.

Secondly, this paragraph would be much easier to understand if you first introduce optical water levels and refer to figures 2 and 3 in the text. Given the importance of the relationship for your results you might want to explain your method in more detail. An important missing detail is whether you only interpolate or also extrapolate beyond the available data range?

P9, Figure 2: refer to the figure in the text, e.g. where you introduce the data sets and in section 3.1

P9, 3.2: The optical water levels should be introduced before P8, L15ff.

P10, L4ff: the part about "shifting gaps" and the ROI is unclear. Do you mean that the Landsat 7 gaps are not always exactly at the same place? Did you choose your ROls such that they never contain no-data pixels? How did you ensure that, given the large amount of Landsat 7 data?

P10, L17: reference for the Otsu method?

P10, L22: How did you decide whether to use a linear or 2nd order polynomial fit?

P10, L25: How did you determine cloud cover?

P11, L2: How much data pairs did you end up with per lake, and how did you select pairs with regard to acquisition dates? I assume you did not always have altimetry and 
shoreline data from the same date (?)

P11, figure 3: c) You might want to colour the dots according to time to check for (and show the readers that there is no) temporal bias. From d), it seems optical water levels are somewhat too high around 2004 and 2015, but too low around 2009?

P12, L9: How did you derive these ROls? Are they drawn manually?

Interactive

comment

P12, L15: regression between the lake area and ..?

P13: As far as I am aware, Strahler's catchment hypsometric model is based on river catchments with a pour point, not endorheic lake catchments as it is the case for the TP. I am not entirely sure what you used this model for (to compute lake water volumes?), but I am not convinced that this is a correct approach. I am also not sure why you need that relationship at all? If you have lake area and lake level time series, you can directly compute volume changes from these?

$\mathrm{P} 14$, Figure 6: It is unclear what the parameters $\mathrm{y}, \mathrm{x}, \mathrm{z}, \mathrm{a}$ and $\mathrm{d}$ represent.

P14, table 3: state nr. of data pairs (optical shoreline position + altimetric lake level) rather than optical data points

Validation

\section{P16, L5: unclear sentence}

P16, L25: the drone GPS tracker alone might not be very accurate, you may easily get a skewed/stretched image composite. Did you use ground control points?

P16, L27: This seems a rather dodgy way to determine the resolution of your image composite.

P17, figure7: which lake? images a) and e) should have the same size/spatial resolution. An overview map would be useful. 
P17, L9ff: extrapolated or interpolated? Provide the parameters and statistical relationship here, maybe even in an additional figure.

P18, figure 8b: add 1:1 line and error bars for the data points

P18, 19: How did you coregistrate the UAV image composite and Landsat image? It seems a spatial shift will completely alter the (relative) shoreline position and thus the basis for your entire analysis: In Figure 9, shifting the shoreline only slightly in e.g. north-south direction will greatly change water/land (sub)pixel counts and thus the basis for the relationship in (b). In my opinion, an error analysis would require several image pairs (UAV and satellite-borne) and a solid coregistration basis, e.g. river/road crossings as clear tie points, or at least a round lake or elongated penninsula rather than a straight shore line.

\section{P18, L7: what do you mean with "concurrent"? What dates?}

P22, L1ff: don't forget the local conditions: ice, snow, wet, dry, muddy shore conditions or also waves greatly affect the water classification result.

\section{Applications}

P22, L10f: Are these your own numbers? How do they compare to previous estimates?

P22, L14ff: mark all lakes mentioned in the text in the map. If they are very close to each other, an extra zoom-in map might be useful.

P23f: restructure section 5 to avoid splitting the Selin Co basin analysis in two sections (5.1 and 5.3). How much of this is new, i.e. has not been published before? How does your dataset make a difference?

P23, L5ff: You mention only the study of Yao et al. (2018). How about other publications? Also, from figure 12 it seems quite clear that the Yao data contains two outliers. Consider using a robust fitting method rather than regular linear regression. 
P24, L10: Depicting intra-annual variation is a strength of your dataset that you might want to emphasize more.

P24, 5.2: Rather than treating the comparison to the LEGOS Hydroweb data as an application case this should be part of the validation section!

P25, L8: "some kind of" bias removal: be more specific. The magnitude of the vertical shift between the two datasets fits to e.g. geoid/ellipsoid height confusion, but the temporal variability of the shift is worrying. Rather than speculating about the cause and assuming that the Hydroweb data is wrong you ought to find the reason for the differences - which may lie in your data processing/method.

P25, L13ff: "reverse relationship" and the conclusion you draw (Hydroweb may "underestimate decreasing trends"): unclear what you mean

P25, figure 15: What are the red/blue shaded areas? (a) compare the series after removing the shift. Sadly, the series (b) and (c) are nowhere discussed. The temporally varying offsets between the series from different data sources should be analysed and removed, or at least explained.

P26, figure 16: again, there seems to be some time-dependent offset between the optical and altimetry-based lake levels, e.g. optical levels are too high around 2005 in the top left panel, and too low around 2005 vs. too high from ca. 2013 in the middle right panel. Can you explain this?

P28, figure 17: What does the blue shaded area show? What data are you showing in these time series? Is the right panel a zoom-in of the left panel?

P28, L17: "Team, 2017": check author name

P29, figure 18: acquisition dates of the images in b) and c)?

P29ff: The entire overflow analysis (lots of new methods introduced) seems to be a study on its own and somewhat out of place in the applications ( results) section of this 
paper.

ESSDD

Conclusions

A short summary of your methods should be provided, in particular the novelty of using shoreline positions from optical data to interpolate between available lake level measurements.

P31, L7: rephrase the sentence to avoid brackets.

P31, L10f: Unclear what you mean. From the comparison you provide currently, I am not yet convinced that your dataset is more correct than the Hydroweb data.

P31, L18: "rigorous uncertainty analysis": As mentioned above, I am not convinced about the theoretical uncertainty exercise you provide.

P31, L25ff: These insights about extrapolating using the derived statistical relationship are very important, but currently not quantified, mentioned or discussed anywhere else in the paper.

Interactive comment on Earth Syst. Sci. Data Discuss., https://doi.org/10.5194/essd-2019-34, 2019. 